\title{
Density Functional Theory Demonstration of Anomeric Effect and Structure: Conformational and Configurational Analysis of $N$-2-(1,4-Dioxane)- $N^{\prime}$-(4-methylbenzenesulfonyl)-O-(4-methylphenoxy) Isourea
}

\author{
Hossein A. Dabbagh, ${ }^{*}$ Ali Reza Najafi Chermahini, and Ali Reza Modarresi-Alam ${ }^{\dagger}$ \\ Department of Chemistry, Isfahan University of Technology, Isfahan 8415483111, Iran. *E-mail: dabbagh@cc.iut.ac.ir \\ 'Department of Chemistry, Sistan \& Baluchestan University, Zahedan, Iran
}

Received May 4, 2005

\begin{abstract}
The conformational, configurtational behavior and the structure of $N$-2-(1,4-Dioxane)- $N^{\prime}-(4-$ methylbenzenesulfonyl)-O-(4-methylphenoxy) isourea 1 has been studied using DFT method. Calculations predict the imidoyl amino group of the dioxane ring prefers axial conformation and that the tosyl and tolyl groups about the $\mathrm{C}=\mathrm{N}$ bond retain $E$ configuration. The anomeric effect controls the population of dioxane ring conformers, and anomers. Intramolecular hydrogen bonds contribute to the stability of $E$ isomers. The computational analysis of $\mathbf{1}$ complements the X-ray findings.
\end{abstract}

Key Words : DFT, Anomeric effect, Conformational analysis, Configurational analysis, Hydrogen bonding

\section{Introduction}

In monosubstituted cyclohexanes, a preference for the equatorial orientation of substituents is generally observed..$^{1-3}$ The lower stability of the axial orientation of a substituent at $\mathrm{C}(1)$ has been attributed to 1,3-diaxial interactions, originating from non-bonded steric repulsions from the two methylene groups at $\mathrm{C}(3)$ and $\mathrm{C}(5) .{ }^{1-5}$ On the other hand, according to theoretical investigations, steric effects of this kind do not seem to exert a significant influence on the equatorial preference of the methyl group and OR substituents. ${ }^{6,7}$ An unexpected increase in the population of the axial conformer has been observed experimentally in monosubstituted cyclohexanes with the $\mathrm{OC}(\mathrm{O}) \mathrm{CH}_{n} \mathrm{~L}_{3-\mathrm{n}}$ substituent $(\mathrm{n}=0,1,2,3$ and $\mathrm{L}=\mathrm{H}, \mathrm{F}, \mathrm{Cl}, \mathrm{Br}){ }^{8-14}$ For these molecules the axial preference (anomeric effect) increases roughly in proportion with the bulk of the CHnL3-n group. ${ }^{13}$ There are many example of anomeric behavior of cyclohexanes and heterocyclohexanes and in the presence of almost constant steric effects, hyperconjugation seems a reasonable explanation. ${ }^{15}$

In monosubstituted cyclohexanes with $\mathrm{OH}$ and $\mathrm{OMe}$ substituents, the equatorial conformer is preferred ${ }^{2}$ an explanation reproduced by $a b$ initio calculations. ${ }^{7}$ The preference for the equatorial conformer is even greater for the Me substituent and all these behaviors fit well with the results of calculations. ${ }^{6,7}$ The marked preference of the equatorial orientation of the Methyl substituent has been attributed fundamentally to hyperconjugative interactions and not to steric effects, and the same conclusion has been reached for cyclohexanol. ${ }^{7}$

Juaristi and coworkers studied anomeric effect in several 1,3 dioxane derivatives and concluded that in compounds with good substitute for hydrogen bonding, axial conformer is prefferd. ${ }^{16}$

Recently, Dabbagh and coworkers studied the structure, conformation of 1,4-dioxane, configuration of the imine group of the imidoyl moiety and the anomeric effect of $\mathbf{1}$ using X-ray crystallographic analysis, Figure $1 .{ }^{17}$

The purpose of the present paper is to establish that the large imidoyl-amino group $\left[\left(4-\mathrm{CH}_{3}-\mathrm{C}_{6} \mathrm{H}_{4}-\mathrm{O}-\mathrm{C}=\mathrm{N}-\mathrm{SO}_{2}-\right.\right.$ $\mathrm{C}_{6} \mathrm{H}_{4}-\mathrm{CH}_{3}-4$ )-NH-] adopts an axial position (the anomeric effect) using the DFT method. Additional aims of this report are to investigate the factors (hydrogen bonds and steric hindrance to anomeric effect) that contribute to this axial preference and to study the conformations and configurations of 1, Figure 1. We believe this system, allows us to shed more light on the phenomenon known as the anomeric effect.

\section{Results and Discussion}

Synthesis. $N$-2-(1,4-Dioxane)-N'-(4-methylbenzenesulfonyl)$O$-(4-methyl phenoxy) isourea 1 was synthesized by the thermal decomposition of $N^{\prime}$-(4-methylbenzenesulfonyl)- $O$ (4-methylphenoxy)imidoyl azide 2 in refluxing dioxane and characterized by ${ }^{1} \mathrm{H}-\mathrm{NMR},{ }^{13} \mathrm{C}-\mathrm{NMR}, \mathrm{COESY}, \mathrm{X}$-ray crystallography, mass spectra analysis, IR and elemental analysis, Figure 1. ${ }^{17}$

Computational details. The relative stability and molecular properties of the structures under investigation were determined by the analytic techniques employing the hybrid density functional method (DFT) with Becke's threeparameter functional for exchange and the Lee, Yang and Parr correlation functional (B3LYP). ${ }^{18,19}$ The adequacy of density functional method for the study of the conformational analysis and anomeric effect has been subject of several studies. ${ }^{20-23}$ All these calculations were carried out on a Pentium IV personal computer by means of GAUSSIAN98W. ${ }^{24}$ The 3-21G* basis set at B3LYP level was selected for calculations and the $3-21 \mathrm{G}^{*}$ optimized geometries were then taken for further optimization with $6-31 \mathrm{G}^{*}$ basis set on 

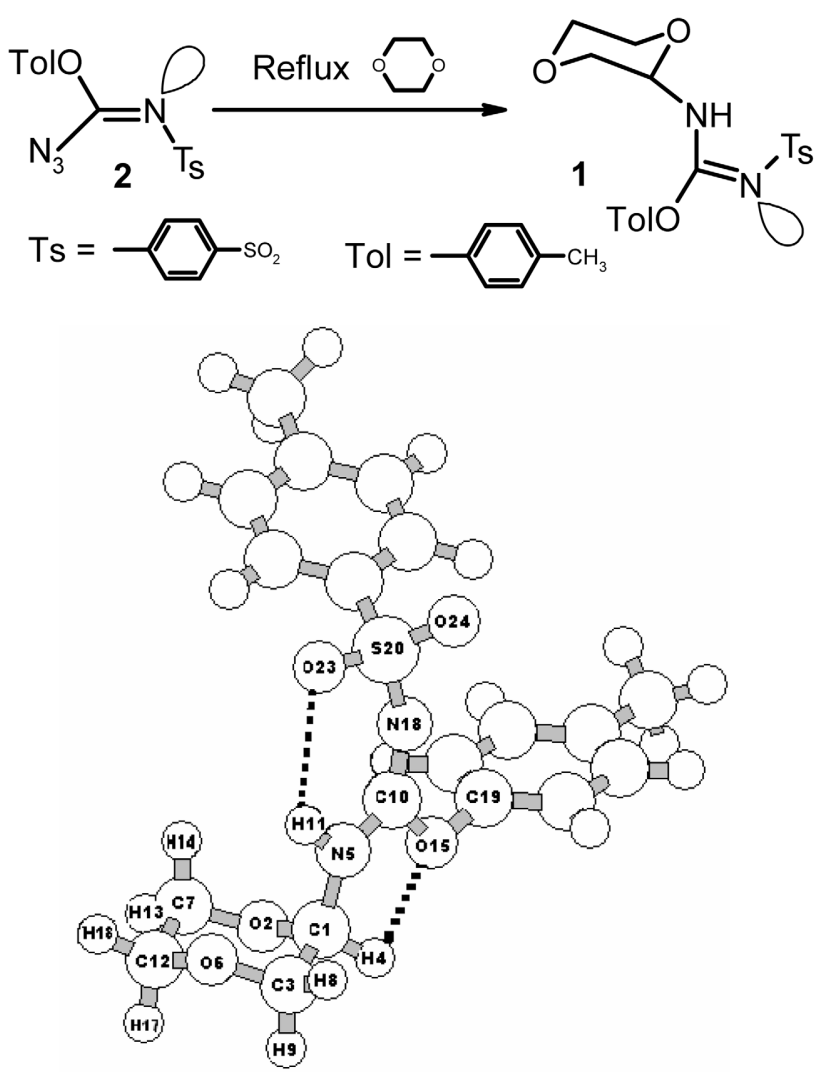

Figure 1. Synthesis and selected numbering of an optimized structure of $\mathbf{1}$. Lines $(\cdots)$ indicate hydrogen-bonds.

density functional theory (B3LYP). To characterize all the optimized geometries, the vibrational frequencies of all conformers were calculated at B3LYP levels.

Geometry and energy. All calculations $6-31 \mathrm{G}^{*}\left(3-21 \mathrm{G}^{*}\right)$ have been consistent in predicting that conformer $1 E_{\text {ax }} \mathbf{A}$ is the most stable conformers in the gas phase, Scheme 1, Tables 1-6, Figure 2.

Initially, we incorrectly predicted the insertion of bulky imidoyl nitrene to favor the equatorial conformer of the dioxane ring (Reverse Anomeric Effect, RAE) ${ }^{25}$ and steric hindrance overcome the anomeric effect, but X-ray and DFT analysis indicate that there is no RAE due to steric hindrance and the bulky imidoyl group retains a proper axial position, which minimizes any repulsion with the hydrogens of the<smiles>ON1CCOC1</smiles>

1EaxA<smiles>ON1CCOC1</smiles>

1EaxB<smiles>CN1CCOC1</smiles>

1EaxC

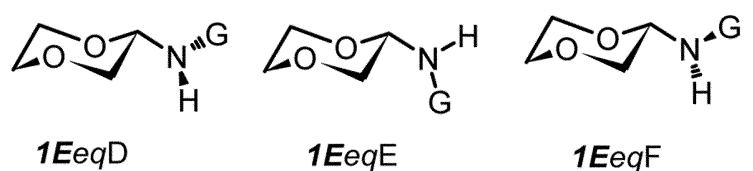

Scheme 1 dioxane ring. The calculated total molecular energy and relative stability of conformers of amino imino group (-NHG) are listed in Table 1. Calculated energy differences between axial and equatorial conformers show that conformer $1 E_{a x} \mathbf{C}$ with bulky immidoyl group in axial position is the least stable conformer (among the $E$ conformers) by 5.06 (4.70) kcal mol ${ }^{-1}$ relative to most stable $\mathbf{1} \boldsymbol{E}_{a x} \mathbf{A}$ conformer. The $\mathbf{1} \boldsymbol{E}_{a x} \mathbf{B}$ conformer with no significant steric repulsion is 4.16 (1.76) $\mathrm{kcal} \mathrm{mol}^{-1}$ less stable than $\boldsymbol{1}_{a x} \mathbf{A}$ conformer. Comparison of $E$ and $Z$ isomers indicates that the $E$ isomers are more stable than the $Z$ isomers. The $Z$ isomers have significant repulsion between lone pairs of phenolic oxygen and sulfonyl oxygenes and no hydrogen bonding, Scheme 2, Table 1 and Figure 2.

Geometrical characterization of compound $\mathbf{1}$ is listed in Tables 2 and 3, Figure 2. The results indeed indicate delocalization of dioxane oxygen lone-pair (anomeric effect). This is in good agreement with the experimental data (X-ray) obtained for $\mathbf{1}^{17}$ and similar to those found in other compounds exhibiting anomeric effect. ${ }^{26-28}$ In addition comparison of the $6-31 \mathrm{G}^{*}\left(3-21 \mathrm{G}^{*}\right)$ geometries for axial and equatorial imidoyl group reveal an increase of C(1)$\mathrm{N}(5)$ bond length, a decrease of $\mathrm{C}(1)-\mathrm{H}(4)$ bond length and widening of $\mathrm{C}(1) \mathrm{O}(2) \mathrm{C}(7)$ bond angle in axial form. Calculations show shorter $\mathrm{C}(1)-\mathrm{O}(2)$ bond length (normal CO bond length, $1.441 \AA)^{29}$ and longer $\mathrm{C}(1)-\mathrm{N}(5)$ bond length (normal C-N bond length, $1.440 \AA$ ). ${ }^{17}$ There is an important through-space interaction between oxygen atoms of sulfonyl group $\left(\mathrm{SO}_{2}\right)$ and hydrogen atom connected to $\mathrm{N}(5)$. Calculations indicate $\mathrm{S}(20)-\mathrm{O}(23)$ bond length is longer than $\mathrm{S}(20)-\mathrm{O}(24)$. This is attributed to hydrogen bonding and/or

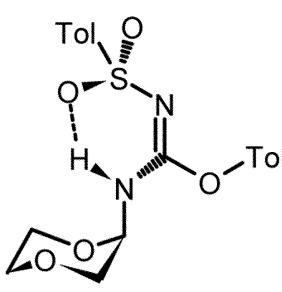

1EaxA

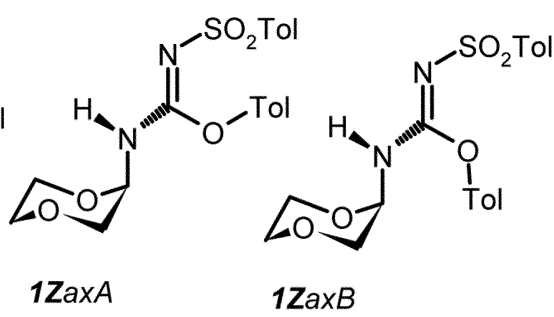

Scheme 2
Table 1. The total and relative energy of 1 calculated by B3LYP/6$31 \mathrm{G}^{*}$ and B3LYP/3-21G*

\begin{tabular}{lcclll}
\hline \multirow{2}{*}{ Entry } & \multicolumn{2}{c}{$3-21 \mathrm{G}^{*}$} & & \multicolumn{2}{c}{$6-31 \mathrm{G}^{*}$} \\
\cline { 2 - 3 } \cline { 5 - 6 } & Total energy $^{a}$ & $\Delta \mathrm{E}^{b}$ & & Total energy $^{a}$ & $\Delta \mathrm{E}^{b}$ \\
\hline $\boldsymbol{E}_{\text {ax }} \mathbf{A}$ & -1612.4766023 & 0.00 & & -1621.0061760 & 0.00 \\
$\boldsymbol{E}_{\text {ax }} \mathbf{B}$ & -1612.4737997 & 1.76 & & -1620.9995499 & 4.16 \\
$\boldsymbol{E}_{\text {ax }} \mathbf{C}$ & -1612.4691182 & 4.70 & & -1620.998103 & 5.06 \\
$\boldsymbol{E}_{\text {eq }} \mathbf{E}$ & -1612.4697992 & 4.26 & & -1621.0014203 & 2.98 \\
$\boldsymbol{E}_{\text {eq }} \mathbf{F}$ & -1612.4712371 & 3.36 & & -1621.0055488 & 0.39 \\
$\boldsymbol{E}_{\text {eq }} \mathbf{D}$ & -1612.4712674 & 3.34 & & -1621.0054548 & 0.45 \\
$\boldsymbol{Z}_{\text {ax }} \mathbf{A}$ & -1612.4592352 & 10.90 & & -1620.9912257 & 9.38 \\
$\boldsymbol{Z}_{\text {ax }} \mathbf{B}$ & -1612.4625023 & 8.85 & & -1620.992696 & 8.46 \\
\hline
\end{tabular}

${ }^{a}$ Total energy in hartree units. ${ }^{b}$ Relative energy in $\mathrm{kcal} / \mathrm{mol}$. 

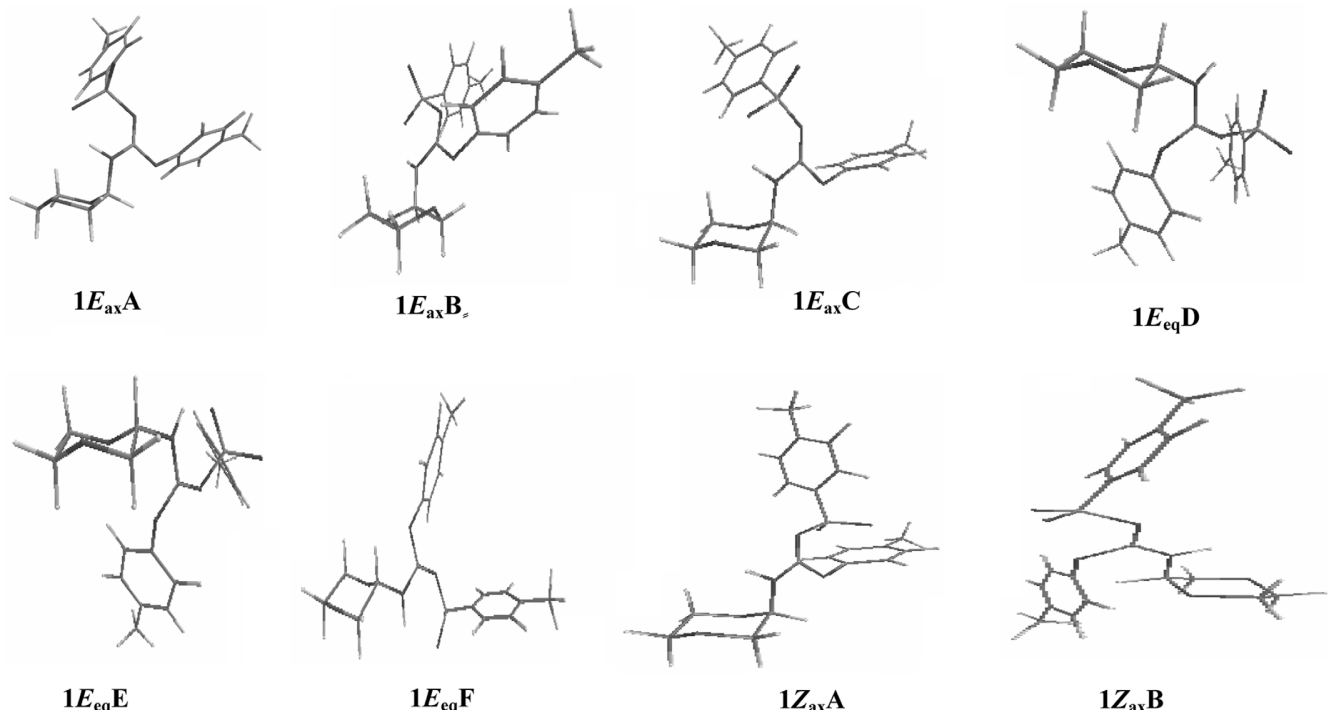

Figure 2. Three-dimensional optimized isomers of $\mathbf{1}$.

Table 2. Selected bond length $(\AA)$, bond angle and torsion angles (deg) of 1 optimized (6-31G*) and calculated by X-ray

\begin{tabular}{|c|c|c|c|c|c|c|c|c|c|}
\hline \multirow{2}{*}{ Entry } & \multicolumn{8}{|c|}{ 6-31G* } & \multirow{2}{*}{$\frac{\text { X-ray }}{\boldsymbol{E}_{\mathrm{ax}} \mathrm{A}}$} \\
\hline & $E_{\text {ax }} \mathrm{A}$ & $E_{\mathrm{ax}} \mathrm{B}$ & $E_{\mathrm{ax}} \mathrm{C}$ & $E_{\mathrm{eq}} \mathrm{D}$ & $E_{\text {eq }} E$ & $E_{\mathrm{eq}} \mathbf{F}$ & $Z_{\mathrm{ax}} \mathrm{A}$ & $Z_{\mathrm{ax}} \mathrm{B}$ & \\
\hline $\mathrm{C} 1-\mathrm{O} 2$ & 1.4259 & 1.4191 & 1.4156 & 1.4303 & 1.4235 & 1.4303 & 1.4259 & 1.4236 & 1.420 \\
\hline C1-H4 & 1.0897 & 1.0953 & 1.0937 & 1.0957 & 1.1009 & 1.0957 & 1.0902 & 1.0903 & $\mathrm{a}$ \\
\hline C1-N5 & 1.4553 & 1.4713 & 1.4678 & 1.4361 & 1.4462 & 1.4361 & 1.4493 & 1.4586 & 1.460 \\
\hline N5-H11 & 1.0205 & 1.0205 & 1.019 & 1.0213 & 1.0203 & 1.0213 & 1.0123 & 1.0152 & $\mathrm{a}$ \\
\hline C1-C3 & 1.5304 & 1.5309 & 1.5459 & 1.532 & 1.5349 & 1.532 & 1.5311 & 1.5309 & 1.530 \\
\hline C19-O15 & 1.4092 & 1.4073 & 1.4015 & 1.4037 & 1.4043 & 1.4036 & 1.4029 & 1.3997 & 1.420 \\
\hline C10-O15 & 1.3437 & 1.3502 & 1.3447 & 1.3494 & 1.3483 & 1.3494 & 1.3523 & 1.3548 & 1.340 \\
\hline N5-C10 & 1.3479 & 1.3544 & 1.3531 & 1.3593 & 1.3593 & 1.3592 & 1.3637 & 1.3706 & 1.331 \\
\hline $\mathrm{S} 20-\mathrm{O} 23$ & 1.4859 & 1.4846 & 1.4867 & 1.4859 & 1.4865 & 1.4859 & 1.4684 & 1.4676 & 1.447 \\
\hline $\mathrm{S} 20-\mathrm{O} 24$ & 1.4665 & 1.4726 & 1.4654 & 1.4664 & 1.4663 & 1.4663 & 1.4715 & 1.4725 & 1.433 \\
\hline C3C1N5 & 108.9694 & 116.079 & 158.823 & 109.9819 & 114.8145 & 109.979 & 108.6913 & 107.845 & 108.2 \\
\hline C1N5C10 & 123.757 & 131.427 & 131.387 & 124.3844 & 126.836 & 124.392 & 125.9688 & 124.059 & 123.0 \\
\hline $\mathrm{C} 1 \mathrm{O} 2 \mathrm{C} 7$ & 112.7268 & 112.903 & 115.548 & 111.4641 & 111.456 & 111.467 & 113.0092 & 113.072 & 115.0 \\
\hline $\mathrm{O} 6 \mathrm{C} 3 \mathrm{C} 1 \mathrm{O} 2$ & -56.5990 & -56.757 & -57.678 & -56.358 & -57.533 & -56.362 & -58.089 & -57.758 & -54.34 \\
\hline
\end{tabular}

${ }^{a}$ Not available.

an interaction between $\mathrm{N}(16)$ lone pair and $\mathrm{S}(20) \sigma^{*}$ (see NBO analysis), Table 2.

Natural bond orbital analysis (NBO). For a further understanding of the conformational preference of $E$ and $Z$ conformers, NBO analysis was carried out at the B3LYP/6$31 \mathrm{G}^{*}$ level of theory. The stabilization energy $\mathrm{E}_{(2)}$ associated with the charge transfer (CT) interactions was obtained subsequently from the second-order perturbative estimates of Fock matrix in the NBO basis. The selected donor acceptor interaction stabilization energy terms may quantitatively account for differences among the selected conformations, Tables 4-6. It is assumed that CT interactions are more important and stabilization energy terms come from filled-empty-orbital hyperconjugative interactions.

There is a strong interaction between nitrogen lone pair $\mathrm{N}(5)$ as donor and $\mathrm{C}(1)-\mathrm{O}(2) \sigma^{*}$ as acceptor for both axial and equatorial conformers, Tables 4-6. However, the $\mathrm{O}(2)$ lone pair as donor and $\mathrm{C}(1)-\mathrm{N}(5)$ as acceptor contribute more to the stability of the axial conformer. This hyperconjugative effect is called endo anomeric effect. The interaction between $\mathrm{O}(2)$ lone pairs as donor and $\mathrm{C}(1)-\mathrm{H}(4)$ $\sigma^{*}$ as acceptor contribute to the stability of equatorial conformers.

NBO analysis also predicted (confirmed by X-ray) ${ }^{17}$ two different bond lengths for $\mathrm{S}(20)-\mathrm{O}(23)$ and $\mathrm{S}(20)-\mathrm{O}(24)$. The hydrogen bonding between $\mathrm{O}(23)$ lone pair and $\mathrm{N}(5)-\mathrm{H}(11)$ $\sigma^{*}$ in $E_{\text {ax }}$ and $E_{\text {eq }}$ isomers and the $n-\sigma^{*}$ interaction between $\mathrm{N}(16)$ lone pair and $\mathrm{S}(20)-\mathrm{O}(23)$ antibonding orbital are presumed to be responsible for this difference and for the stability of the $E$ isomers over the $Z$ isomer. Hydrogen bonding does not contribute to the stability of the $Z$ isomers. Finally, DFT calculations show a weak interaction between $\mathrm{O}(15)$ lone pair and $\mathrm{C}(1)-\mathrm{H}(4) \sigma^{*}$ in $\mathbf{1} \boldsymbol{E}_{\mathrm{ax}} \mathbf{A}$ conformer. This interaction is in excellent agreement with experimental 
Table 3. Selected bond length $(\AA)$, bond angle and torsion angles (deg) of 1 optimized by $3-21 \mathrm{G}^{*}$

\begin{tabular}{|c|c|c|c|c|c|c|c|c|}
\hline \multirow{2}{*}{ Entry } & \multicolumn{8}{|c|}{$3-21 G^{*}$} \\
\hline & $E_{\text {ax }} \mathbf{A}$ & $E_{\mathrm{ax}} \mathrm{B}$ & $E_{\mathrm{ax}} \mathrm{C}$ & $E_{\text {eq }} \mathbf{D}$ & $E_{\text {eq }} \mathbf{E}$ & $E_{\text {eq }} \mathbf{F}$ & $Z \mathbf{a x A}$ & $Z$ axB \\
\hline $\mathrm{C} 1-\mathrm{O} 2$ & 1.4674 & 1.4191 & 1.4568 & 1.472 & 1.4765 & 1.4718 & 1.4687 & 1.4652 \\
\hline $\mathrm{C} 1-\mathrm{H} 4$ & 1.0864 & 1.0953 & 1.0927 & 1.0917 & 1.0975 & 1.0918 & 1.0867 & 1.0873 \\
\hline C1-N5 & 1.4567 & 1.3544 & 1.4680 & 1.4392 & 1.4473 & 1.4392 & 1.451 & 1.4558 \\
\hline N5-H11 & 1.028 & 1.0205 & 1.0295 & 1.0302 & 1.0311 & 1.0302 & 1.0172 & 1.0195 \\
\hline $\mathrm{C} 1-\mathrm{C} 3$ & 1.5288 & 1.5309 & 1.5551 & 1.5323 & 1.5340 & 1.5323 & 1.5302 & 1.530 \\
\hline C19-O15 & 1.4162 & 1.4328 & 1.4205 & 1.4168 & 1.4290 & 1.4167 & 1.4273 & 1.4342 \\
\hline $\mathrm{C} 10-\mathrm{O} 15$ & 1.3662 & 1.3743 & 1.3598 & 1.3642 & 1.3819 & 1.3641 & 1.3897 & 1.393 \\
\hline N5-C10 & 1.3578 & 1.3587 & 1.3574 & 1.3531 & 1.3606 & 1.3604 & 1.3624 & 1.3619 \\
\hline C3-H8 & 1.0976 & 1.1016 & 1.0994 & 1.0912 & 1.0914 & 1.0912 & 1.0968 & 1.0916 \\
\hline C3-H9 & 1.0909 & 1.0878 & 1.0892 & 1.1006 & 1.0921 & 1.1006 & 1.0912 & 1.0969 \\
\hline $\mathrm{S} 20-\mathrm{O} 23$ & 1.488 & 1.4846 & 1.4886 & 1.4879 & 1.4901 & 1.4881 & 1.4705 & 1.4748 \\
\hline $\mathrm{S} 20-\mathrm{O} 24$ & 1.4634 & 1.4726 & 1.4622 & 1.4624 & 1.4616 & 1.4624 & 1.467 & 1.4657 \\
\hline C3C1N5 & 107.0661 & 116.079 & 129.76 & 110.0977 & 114.5191 & 110.1027 & 106.8017 & 106.7226 \\
\hline $\mathrm{C} 1 \mathrm{~N} 5 \mathrm{C} 10$ & 123.8553 & 131.43 & 123.68 & 124.2891 & 124.80 & 124.2808 & 125.4332 & 126.8727 \\
\hline $\mathrm{C} 1 \mathrm{O} 2 \mathrm{C} 7$ & 110.3536 & 112.903 & 112.99 & 109.9114 & 109.46 & 111.4860 & 110.8754 & 110.6822 \\
\hline $\mathrm{O} 6 \mathrm{C} 3 \mathrm{C} 1 \mathrm{O} 2$ & -59.3412 & -58.9656 & -58.0709 & -59.6924 & -59.0991 & -59.6849 & -64.2712 & -64.0759 \\
\hline
\end{tabular}

Table 4. Selected donor-acceptor natural bond orbital interactions and their second-order perturbation stabilization energies, $\Delta \mathrm{E}(2)(\mathrm{kcal}$ $\mathrm{mol}^{-1}$ ), calculated at the B3LYP/6-31G* level for axial conformers

\begin{tabular}{|c|c|c|c|c|c|}
\hline \multirow{2}{*}{ Donor NBO } & \multirow{2}{*}{ Acceptor NBO } & \multirow{2}{*}{ Interactions } & \multicolumn{3}{|c|}{$\Delta \mathrm{E}(2)$} \\
\hline & & & $1 E_{\text {ax }} \mathrm{A}$ & $1 E_{a x} B$ & $1 \mathrm{E}_{\mathrm{ax}} \mathrm{C}$ \\
\hline $\mathrm{BD}(1) \mathrm{C} 1-\mathrm{N} 5$ & $\mathrm{BD}^{*}(1) \mathrm{C} 10-\mathrm{N} 16$ & $\sigma-\sigma^{*}$ & 4.02 & 3.52 & 3.06 \\
\hline $\mathrm{BD}(1) \mathrm{C} 1-\mathrm{H} 4$ & $\mathrm{BD}^{*}(1) \mathrm{O} 2-\mathrm{C} 7$ & $\sigma-\sigma^{*}$ & 3.53 & 3.60 & 2.59 \\
\hline $\mathrm{BD}$ (1) $\mathrm{C} 1-\mathrm{H} 4$ & $\mathrm{BD}^{*}(1) \mathrm{C} 3-\mathrm{O} 6$ & $\sigma-\sigma^{*}$ & 3.49 & 3.52 & 3.26 \\
\hline $\mathrm{BD}(1) \mathrm{O} 2-\mathrm{C} 7$ & $\mathrm{BD}^{*}(1) \mathrm{C} 1-\mathrm{H} 4$ & $\sigma-\sigma^{*}$ & 1.01 & 0.95 & 1.89 \\
\hline $\mathrm{BD}(10 \mathrm{~N} 5-\mathrm{C} 10$ & $\mathrm{BD}^{*}(1) \mathrm{C} 1-\mathrm{N} 5$ & $\sigma-\sigma^{*}$ & 1.15 & 1.17 & 1.61 \\
\hline BD (1) N5-C10 & BD*(1) O15-C19 & $\sigma-\sigma^{*}$ & 2.18 & 2.41 & 1.85 \\
\hline BD (1) N5-H11 & $\mathrm{BD}^{*}$ (1) $\mathrm{C} 10-\mathrm{O} 15$ & $\sigma-\sigma^{*}$ & 4.72 & 4.28 & 3.80 \\
\hline $\mathrm{BD}$ (1) C10-N16 & $\mathrm{BD} *(1) \mathrm{C} 1-\mathrm{N} 5$ & $\sigma-\sigma^{*}$ & 1.76 & 1.67 & 1.81 \\
\hline BD (1) C10-N16 & BD*(1) N5-C10 & $\sigma-\sigma^{*}$ & 1.66 & 1.70 & 2.10 \\
\hline $\mathrm{LP}(1) \mathrm{O} 2$ & $\mathrm{BD}^{*}(1) \mathrm{C} 1-\mathrm{C} 3$ & $n-\sigma^{*}$ & 2.13 & 2.07 & 1.67 \\
\hline $\mathrm{LP}(1) \mathrm{O} 2$ & BD*(1) C1-N5 & $n-\sigma^{*}$ & 0.79 & 0.90 & 1.10 \\
\hline $\mathrm{LP}(2) \mathrm{O} 2$ & $\mathrm{BD}^{*}(1) \mathrm{C} 1-\mathrm{N} 5$ & $n-\sigma^{*}$ & 11.29 & 10.85 & 10.47 \\
\hline $\mathrm{LP}(2) \mathrm{O} 2$ & $\mathrm{BD}^{*}(1) \mathrm{C} 1-\mathrm{C} 3$ & $n-\sigma^{*}$ & 4.12 & 4.06 & 4.87 \\
\hline LP (1) N5 & $\mathrm{BD}^{*}(1) \mathrm{C} 1-\mathrm{O} 2$ & $n-\sigma^{*}$ & 13.80 & 12.36 & 5.69 \\
\hline LP (1) N5 & $\mathrm{BD}^{*}(1) \mathrm{C} 1-\mathrm{C} 3$ & $n-\sigma^{*}$ & 1.45 & - & 5.62 \\
\hline LP (1) N5 & $\mathrm{BD}^{*}(1) \mathrm{C} 1-\mathrm{H} 4$ & $n-\sigma^{*}$ & 0.61 & 3.46 & 0.56 \\
\hline LP (1) N5 & BD*(1) C10-N16 & $n-\sigma^{*}$ & 61.42 & 62.37 & 39.89 \\
\hline LP (1) O6 & $\mathrm{BD}^{*}(1) \mathrm{C} 1-\mathrm{C} 3$ & $n-\sigma^{*}$ & 1.54 & 1.57 & 1.28 \\
\hline LP (2) O6 & $\mathrm{BD}^{*}(1) \mathrm{C} 1-\mathrm{C} 3$ & $n-\sigma^{*}$ & 6.10 & 5.97 & 6.10 \\
\hline LP (1) O15 & $\mathrm{BD}^{*}(1) \mathrm{N} 5-\mathrm{C} 10$ & $n-\sigma^{*}$ & 1.53 & 1.53 & 0.77 \\
\hline LP (1) O15 & $\mathrm{BD}^{*}(1) \mathrm{C} 10-\mathrm{N} 16$ & $n-\sigma^{*}$ & 6.29 & 6.40 & 6.14 \\
\hline LP (2) O15 & $\mathrm{BD}^{*}$ (1) C10-N16 & $n-\sigma^{*}$ & 36.68 & 36.40 & 4.93 \\
\hline LP (1) O23 & BD*(1) N5-H11 & $n-\sigma^{*}$ & 1.38 & 8.17 & - \\
\hline LP (2) O23 & BD*(1) N5-H11 & $n-\sigma^{*}$ & 4.51 & 6.52 & 2.49 \\
\hline LP (1) N16 & $\mathrm{BD} *(1) \mathrm{S} 20-\mathrm{O} 24$ & $n-\sigma^{*}$ & 16.36 & 17.58 & 3.01 \\
\hline LP (1) N16 & $\mathrm{BD}^{*}(1) \mathrm{S} 20-\mathrm{O} 23$ & $n-\sigma^{*}$ & 5.23 & 4.61 & 0.75 \\
\hline
\end{tabular}

E, denotes the stabilization energy; $\mathrm{BD}$, denotes bonding orbital; $\mathrm{BD}^{*}$, denotes antibonding orbital; Lp, denotes lone-pair. For BD and BD*, (1) and (2) denote -orbital and -orbital, respectively. For Lp (1) and (2) denote the first and second lone pair electron, respectively. 
Table 5. Selected donor-acceptor natural bond orbital interactions and their second-order perturbation stabilization energies, $\Delta E(2)(\mathrm{kcal}$ $\mathrm{mol}^{-1}$ ), calculated at the B3LYP/6-31G* level for equatorial conformers

\begin{tabular}{|c|c|c|c|c|c|}
\hline \multirow{2}{*}{ Donor NBO } & \multirow{2}{*}{ Acceptor NBO } & \multirow{2}{*}{ Interactions } & \multicolumn{3}{|c|}{$\Delta E(2)$} \\
\hline & & & $1 E_{\mathrm{eq}} \mathrm{D}$ & $1 E_{\mathrm{eq}} \mathbf{E}$ & $1 E_{\mathrm{eq}} \mathrm{F}$ \\
\hline $\mathrm{BD}(1) \mathrm{C} 1-\mathrm{N} 5$ & $\mathrm{BD}^{*}(1) \mathrm{O} 2-\mathrm{C} 7$ & $\sigma-\sigma^{*}$ & 1.46 & 1.51 & 1.45 \\
\hline $\mathrm{BD}$ (1) O2-C7 & $\mathrm{BD} *(1) \mathrm{C} 1-\mathrm{N} 5$ & $\sigma-\sigma^{*}$ & 2.05 & 2.01 & 2.03 \\
\hline $\mathrm{BD}(1) \mathrm{N} 5-\mathrm{C} 10$ & $\mathrm{BD}^{*}(1) \mathrm{C} 1-\mathrm{N} 5$ & $\sigma-\sigma^{*}$ & 1.05 & 1.05 & 1.11 \\
\hline BD (1) N5-C10 & $\mathrm{BD}^{*}(1) \mathrm{C} 10-\mathrm{N} 16$ & $\sigma-\sigma^{*}$ & 1.90 & 1.72 & 1.56 \\
\hline BD (1) N5-C10 & $\mathrm{BD}^{*}(1) \mathrm{O} 15-\mathrm{C} 19$ & $\sigma-\sigma^{*}$ & 2.18 & 2.06 & 2.19 \\
\hline BD (1) N5-C10 & BD*(1) N16-S20 & $\sigma-\sigma^{*}$ & 1.73 & 0.97 & - \\
\hline BD (1) N5-H11 & BD*(1) C10-O15 & $\sigma-\sigma^{*}$ & 3.98 & 4.45 & 4.00 \\
\hline BD (1) N5-H11 & BD*(1) C10-N16 & $\sigma-\sigma^{*}$ & 0.69 & 0.51 & 0.74 \\
\hline BD (1) C10-N16 & $\mathrm{BD}^{*}(1) \mathrm{C} 1-\mathrm{N} 5$ & $\sigma-\sigma^{*}$ & 1.68 & 1.75 & 1.64 \\
\hline $\mathrm{BD}$ (1) C10-N16 & BD* (1) S20-O24 & $\sigma-\sigma^{*}$ & 7.95 & 7.25 & 9.31 \\
\hline BD (1) C10-N16 & $\mathrm{BD}^{*}(1) \mathrm{S} 20-\mathrm{O} 23$ & $\sigma-\sigma^{*}$ & 2.05 & 0.54 & 1.61 \\
\hline $\mathrm{LP}(1) \mathrm{O} 2$ & $\mathrm{BD} *(1) \mathrm{C} 1-\mathrm{C} 3$ & $n-\sigma^{*}$ & 0.79 & 1.18 & 0.74 \\
\hline $\mathrm{LP}(1) \mathrm{O} 2$ & $\mathrm{BD}^{*}(1) \mathrm{C} 1-\mathrm{H} 4$ & $n-\sigma^{*}$ & 1.23 & 0.93 & 1.27 \\
\hline $\mathrm{LP}(1) \mathrm{O} 2$ & $\mathrm{BD}^{*}(1) \mathrm{C} 1-\mathrm{N} 5$ & $n-\sigma^{*}$ & 2.45 & 2.54 & 2.40 \\
\hline $\mathrm{LP}(2) \mathrm{O} 2$ & $\mathrm{BD} *(1) \mathrm{C} 1-\mathrm{C} 3$ & $n-\sigma^{*}$ & 5.60 & 5.20 & 5.60 \\
\hline $\mathrm{LP}(2) \mathrm{O} 2$ & $\mathrm{BD}^{*}(1) \mathrm{C} 1-\mathrm{H} 4$ & $n-\sigma^{*}$ & 4.72 & 5.43 & 4.62 \\
\hline LP (1) N5 & $\mathrm{BD}^{*}(1) \mathrm{C} 1-\mathrm{O} 2$ & $n-\sigma^{*}$ & 13.99 & 13.03 & 14.11 \\
\hline LP (1) N5 & $\mathrm{BD}^{*}(1) \mathrm{C} 1-\mathrm{C} 3$ & $n-\sigma^{*}$ & 1.21 & 3.72 & 1.09 \\
\hline LP (1) N5 & $\mathrm{BD}^{*}(1) \mathrm{C} 10-\mathrm{N} 16$ & $n-\sigma^{*}$ & 47.38 & 52.24 & 49.80 \\
\hline LP (1) O6 & $\mathrm{BD}^{*}(1) \mathrm{C} 1-\mathrm{C} 3$ & $n-\sigma^{*}$ & 1.20 & 1.11 & 1.19 \\
\hline LP (2) O6 & $\mathrm{BD} *(1) \mathrm{C} 1-\mathrm{C} 3$ & $n-\sigma^{*}$ & 6.03 & 6.36 & 5.99 \\
\hline LP (2) O6 & $\mathrm{BD}^{*}(1) \mathrm{C} 1-\mathrm{N} 5$ & $n-\sigma^{*}$ & 1.00 & 0.99 & 1.00 \\
\hline LP (1) O15 & BD*(1) C10-N16 & $n-\sigma^{*}$ & 7.61 & 7.67 & 7.13 \\
\hline LP (2) O15 & $\mathrm{BD}^{*}(1) \mathrm{C} 10-\mathrm{N} 16$ & $n-\sigma^{*}$ & 37.01 & 32.46 & 35.05 \\
\hline LP (1) O23 & BD*(1) N5-H11 & $n-\sigma^{*}$ & 2.39 & 2.87 & 3.54 \\
\hline LP (2) O23 & BD*(1) N5-H11 & $n-\sigma^{*}$ & 6.20 & 5.89 & 5.42 \\
\hline LP (1) N16 & $\mathrm{BD}^{*}$ (1) S20-O24 & $n-\sigma^{*}$ & 12.68 & 13.65 & 10.26 \\
\hline LP (1) N16 & $\mathrm{BD}^{*}(1) \mathrm{S} 20-\mathrm{O} 23$ & $n-\sigma^{*}$ & 0.93 & 0.68 & 0.75 \\
\hline
\end{tabular}

E, denotes the stabilization energy; BD, denotes bonding orbital; BD*, denotes antibonding orbital; Lp, denotes lone-pair. For BD and BD*, (1) and (2) denote -orbital and -orbital, respectively. For Lp (1) and (2) denote the first and second lone pair electron, respectively<smiles>[O][S@@]1(O)=N[C]N(C(=O)O[Ge])C(=O)N=1</smiles>

Scheme 4

data. $^{17}$

Tables 4 and 5 there are two strong interactions between $\mathrm{O}(15)$ and $\mathrm{N}(5)$ lone pairs and $\mathrm{C}(10)-\mathrm{N}(16) \sigma^{*}$. In addition, $\mathrm{O}(15)-\mathrm{C}(10)$ bond length is shorter than $\mathrm{O}(15)-\mathrm{C}(19)$ bond for all conformers and $\mathrm{N}(5)-\mathrm{C}(10)$ bond length is approximately equal to a $\mathrm{N}\left(\mathrm{sp}^{2}\right)-\mathrm{C}\left(\mathrm{sp}^{2}\right) \cdot{ }^{17}$ The $s$-cis conformation of $\mathrm{S}(20)-\mathrm{O}(23)$ and $\mathrm{C}(10)-\mathrm{N}(16)$ bonds and $\mathrm{S}(20)-\mathrm{O}(23) \mathrm{H}(11)-\mathrm{N}(5)$ hydrogen bond form a stable six member ring, Scheme 4.

\section{Conclusion}

The anomeric effect (hypercojugative effect) plays a major role for the axial preference of the imidoyl amino group $\mathrm{HN}$ $\mathrm{G}\left(\mathrm{G}=4-\mathrm{Me}-\mathrm{C}_{6} \mathrm{H}_{4}-\mathrm{O}-\mathrm{C}=\mathrm{N}-\mathrm{SO}_{2}-\mathrm{C}_{6} \mathrm{H}_{4}-\mathrm{Me}-p\right)$. Energetically, DFT calculation predicts $1 E_{\text {ax }} \mathbf{A}$ isomer as the most stable conformer. The endo anomeric effect, intramolecular hydrogen bonding and steric repulsion of bulky HN-G group control population of conformer and/or configurations (in excellent agreement with X-ray findings). Geometrically, anomeric effect is revealed from shortening of $\mathrm{C}(1)-\mathrm{O}(2)$, increase of $\mathrm{C}(1)-\mathrm{N}(5)$ bond length and widening of $\mathrm{C}(1) \mathrm{O}(2) \mathrm{C}(7)$ angle. All $E$ isomers are more stable than $Z$ isomers (due to steric effects and hydrogen bonding). 
Table 6. Selected donor-acceptor natural bond orbital interactions and their second-order perturbation stabilization energies, $\Delta E$ (2) $\left(\mathrm{kcal} \mathrm{mol}^{-1}\right)$, calculated at the B3LYP/6-31G* level for $Z$ isomers

\begin{tabular}{|c|c|c|c|c|}
\hline \multirow{2}{*}{ Donor NBO } & \multirow{2}{*}{ Acceptor NBO } & \multirow{2}{*}{ Interactions } & \multicolumn{2}{|c|}{$\Delta E(2)$} \\
\hline & & & $1 Z_{\mathrm{ax}} \mathrm{A}$ & $1 Z_{\mathrm{ax}} \mathrm{B}$ \\
\hline $\mathrm{BD}(1) \mathrm{C} 1-\mathrm{C} 3$ & BD* N5-C10 & $\sigma-\sigma^{*}$ & 3.15 & 3.30 \\
\hline $\mathrm{BD}(1) \mathrm{C} 1-\mathrm{N} 5$ & $\mathrm{BD} * \mathrm{C} 10-\mathrm{N} 16$ & $\sigma-\sigma^{*}$ & 3.42 & 3.50 \\
\hline $\mathrm{BD}$ (1) $\mathrm{C} 3-\mathrm{O} 6$ & $\mathrm{BD} * \mathrm{C} 1-\mathrm{H} 4$ & $\sigma-\sigma^{*}$ & 1.03 & 0.89 \\
\hline BD (1) N5-H11 & $\mathrm{BD} * \mathrm{C} 1-\mathrm{H} 4$ & $\sigma-\sigma^{*}$ & 1.67 & 1.44 \\
\hline $\mathrm{BD}(1) \mathrm{N} 5-\mathrm{C} 10$ & $\mathrm{BD} * \mathrm{C} 1-\mathrm{C} 3$ & $\sigma-\sigma^{*}$ & 0.84 & 0.98 \\
\hline BD (1) N5-C10 & $\mathrm{BD} * \mathrm{C} 1-\mathrm{N} 5$ & $\sigma-\sigma^{*}$ & 1.06 & 1.20 \\
\hline BD (1) N5-C10 & $\mathrm{BD} * \mathrm{C} 10-\mathrm{N} 16$ & $\sigma-\sigma^{*}$ & 1.74 & 2.05 \\
\hline BD (1) N5-C10 & BD*N16-S20 & $\sigma-\sigma^{*}$ & 2.98 & 2.94 \\
\hline $\mathrm{BD}$ (1) C10-N16 & BD* N16 S20 & $\sigma-\sigma^{*}$ & 0.85 & 0.70 \\
\hline $\mathrm{BD}$ (1) C10-N16 & $\mathrm{BD} * \mathrm{~S} 20-\mathrm{C} 25$ & $\sigma-\sigma^{*}$ & 1.07 & 0.93 \\
\hline $\mathrm{BD}$ (1) C10-N16 & $\mathrm{BD} *(1) \mathrm{C} 10-\mathrm{N} 5$ & $\sigma-\sigma^{*}$ & 1.70 & 1.63 \\
\hline $\mathrm{BD}$ (1) C10-N16 & $\mathrm{BD}^{*}(1) \mathrm{N} 5-\mathrm{C} 1$ & $\sigma-\sigma^{*}$ & 1.83 & 1.64 \\
\hline LP (1) O15 & $\mathrm{BD}^{*}(1) \mathrm{C} 1-\mathrm{H} 4$ & $n-\sigma^{*}$ & 0.67 & 0.50 \\
\hline LP (1) $\mathrm{O} 15$ & $\mathrm{BD}^{*}(1) \mathrm{C} 10-\mathrm{N} 16$ & $n-\sigma^{*}$ & 1.76 & 4.99 \\
\hline $\mathrm{LP}(2) \mathrm{O} 15$ & $\mathrm{BD}^{*}(1) \mathrm{N} 5-\mathrm{C} 10$ & $n-\sigma^{*}$ & 40.06 & 34.93 \\
\hline LP (1) N 16 & $\mathrm{BD}^{*}(1) \mathrm{C} 10-\mathrm{O} 15$ & $n-\sigma^{*}$ & 19.36 & 21.08 \\
\hline LP (1) N 16 & $\mathrm{BD} *(1) \mathrm{C} 10-\mathrm{N} 5$ & $n-\sigma^{*}$ & 3.30 & 3.36 \\
\hline LP (1) N 16 & $\mathrm{BD}^{*}(1) \mathrm{S} 20-\mathrm{O} 23$ & $n-\sigma^{*}$ & 2.63 & 2.61 \\
\hline LP (1) N 16 & $\mathrm{BD}^{*}(1) \mathrm{S} 20-\mathrm{O} 24$ & $n-\sigma^{*}$ & 4.61 & 3.89 \\
\hline LP (1) N 5 & $\mathrm{BD}^{*}(1) \mathrm{C} 10-\mathrm{N} 16$ & $n-\sigma^{*}$ & 56.36 & 57.65 \\
\hline LP (1) N 5 & $\mathrm{BD}^{*}(1) \mathrm{C} 1-\mathrm{O} 2$ & $n-\sigma^{*}$ & 15.22 & 16.39 \\
\hline LP (1) N 5 & $\mathrm{BD}^{*}(1) \mathrm{C} 1-\mathrm{C} 3$ & $n-\sigma^{*}$ & 1.34 & 1.38 \\
\hline LP (1) N 5 & $\mathrm{BD}^{*}(1) \mathrm{C} 1-\mathrm{H} 4$ & $n-\sigma^{*}$ & 1.07 & 1.30 \\
\hline $\mathrm{LP}(1) \mathrm{O} 2$ & $\mathrm{BD}^{*}(1) \mathrm{C} 1-\mathrm{C} 3$ & $n-\sigma^{*}$ & 2.18 & 2.31 \\
\hline $\mathrm{LP}(1) \mathrm{O} 2$ & $\mathrm{BD}^{*}(1) \mathrm{C} 1-\mathrm{H} 4$ & $n-\sigma^{*}$ & 1.30 & 0.94 \\
\hline $\mathrm{LP}(2) \mathrm{O} 2$ & $\mathrm{BD}^{*}(1) \mathrm{C} 1-\mathrm{N} 5$ & $n-\sigma^{*}$ & 11.39 & 11.50 \\
\hline $\mathrm{LP}(2) \mathrm{O} 2$ & $\mathrm{BD}^{*}(1) \mathrm{C} 1-\mathrm{C} 3$ & $n-\sigma^{*}$ & 4.03 & 3.24 \\
\hline LP (1) O 6 & $\mathrm{BD}^{*}(1) \mathrm{C} 1-\mathrm{C} 3$ & $n-\sigma^{*}$ & 1.44 & 1.44 \\
\hline LP (2) O 6 & $\mathrm{BD}^{*}(1) \mathrm{C} 1-\mathrm{C} 3$ & $n-\sigma^{*}$ & 6.08 & 5.84 \\
\hline
\end{tabular}

$\mathrm{E}$, denotes the stabilization energy; $\mathrm{BD}$, denotes bonding orbital; $\mathrm{BD}^{*}$, denotes antibonding orbital; Lp, denotes lone-pair. For BD and $\mathrm{BD}^{*},(1)$ and (2) denote -orbital and -orbital, respectively. For Lp (1) and (2) denote the first and second lone pair electron, respectively

Acknowledgments. This work was supported by Isfahan University of Technology Graduate Program Council and Research Council Grant IUT-1CHH831. We would like to thank Dr. Hassan Sabzian for many valuable discussions and contributions.

\section{References}

1. Eliel, E. L.; Wilen, S. H. Stereochemistry of Organic Compounds; Wiley: New York, 1994.
2. Bushweller, C. H. Stereochemistry of Cyclohexane and Substituted Cyclohexanes. Substituted A Values in Conformational Behavior of Six-Membered Ring Analysis, Dynamics and Stereoelectronic Effects; Juaristi, E., Ed.; VHC/Wiley: New York, 1995; Chapter 2.

3. Eliel, E. L.; Allinger, N. L.; Angyal, S. J. Conformational Analysis; Wiley: New York, 1965.

4. Winestein, S.; Holness, N. J. J. Am. Chem. Soc. 1955, 55, 5562.

5. (a) Hirsch, J. A. Top Stereochem. 1967, 1, 199. (b) Jensen, F. R.; Bushweller, C. H. Adv. Alicycl. Chem. 1971, 3, 139.

6. Wiberg, K. B.; Hammer, J. D.; Castejon, H.; Bailey, W. F.; De Leon, E.; Jarrett, R. M. J. Org. Chem. 1999, 64, 2085.

7. Salzner, U.; Schleyer, P. V. R. J. Org. Chem. 1994, 59, 2138.

8. Borsdorf, R.; Muller, R.; Tenner, R.; Kleinpeter, E. Z. Chem. 1976, 16, 106

9. Borsdorf, R.; Kleinpeter, E.; Arnold, M. Z. Chem. 1977, 17, 378.

10. Borsdorf, R.; Kleinpeter, E.; Meinel, C.; Lenk, D. Z. Chem. 1978, 18, 185.

11. Kleinpeter, E.; Taddei, F. J. Mol. Struct. 2002, 585, 223.

12. Kleinpeter, E.; Taddei, F. J. Mol. Struct. 2004, 683, 29.

13. Kleinpeter, E.; Taddei, F.; Wacker, P. Chem. Eur. J. 2003, 9, 1360.

14. Lewis, B. E.; Schramm, V. L. J. Am. Chem. Soc. 2001, 123, 1327.

15. Koler, H.; Tschierske, C.; Zaschke, H.; Kleinpeter, E. Tetrahedron 1990, 46, 4241.

16. Juaristi, E.; Rosquete-Pina, G. A. Pure Appl. Chem. 2003, 75, 589

17. Dabbagh, H. A.; Modarresi-Alam, A.; Tadjarodi, A.; Taeb, A. Tetrahedron 2002, 58, 2621.

18. Becke, A. D. J. Chem. Phys. 1993, 98, 5648.

19. Lee, C.; Yang, W.; Parr, R. G. Phys. Rev. B. 1988, 37, 785.

20. Yeunga, G. F. C.; Setiadia, D. H.; Chass, G. A.; Imre G. C. J. Mol. Struct. (Theochem) 2003, 666-667, 393-396.

21. Jiang, Y.; Cole, R. B. J. Am. Soc. Mass. Spectrom. 2005, 16, 60

22. Tvaroska, I.; Carver, J. P. Carbohydrate Research, 1998, 309, 1.

23. Tvaroska, I.; André, I.; Carver, J. P. J. Mol. Struct. (Theochem) 1999, 469, 103.

24. Frisch, M. J.; Trucks, G. W.; Schlegel, H. B.; Scuseria, G. E.; Robb, M. A.; Cheeseman, J. R.; Zakrzewski, V. G.; Montgomery J. A., Jr.; Stratmann, R. E.; Burant, J. C.; Dapprich, S.; Millam, J. M.; Daniels, A. D.; Kudin, K. N.; Strain, M. C.; Farkas, O.; Tomasi, J.; Barone, V.; Cossi, M.; Cammi, R.; Mennucci, B.; Pomelli, C.; Adamo, C.; Clifford, S.; Ochterski, J.; Petersson, G. A.; Ayala, P. Y.; Cui, Q.; Morokuma, K.; Malick, D. K.; Rabuck, A. D.; Raghavachari, K.; Foresman, J. B.; Cioslowski, J.; Ortiz, J. V.; Baboul, A. G.; Stefanov, B. B.; Liu, G.; Liashenko, A.; Piskorz, P.; Komaromi, I.; Gomperts, R.; Martin, R. L.; Fox, D. J.; Keith, T.; Al-Laham, M. A.; Peng, C. Y.; Nanayakkara, A.; Gonzalez, C.; Challacombe, M.; Gill, P. M. W.; Johnson, B.; Chen, W.; Wong, M. W.; Andres, J. L.; Head-Gordon, M.; Replogle, E. S.; Pople, J. A. GAUSSIAN98, Revision A.7; Gaussian: Pittsburgh, PA, 1998.

25. Perrin, C. L. Pure Appl. Chem. 1995, 67, 719.

26. (a) Perrin, C. L.; Fabian, M. A.; Brunckova, J.; Ohta, B. K. J. Am. Chem. Soc. 1999, 121, 6911. (b) Sakurai, S.; Meinander, N.; Morris, K.; Laane, J. J. Am. Chem. Soc. 1999, 121, 5056.

27. Omoto, K.; Marusaki, K.; Hirao, H.; Imade, M.; Fujimoto, H. $J$. Phys. Chem. A 2000, 104, 6499.

28. Tvaroka, I. J. Phys. Chem. A 2000, 100, 11305.

29. Juaristi, E.; Cuevas, G. Tetrahedron 1992, 48, 5019, and references therein. 